\title{
La Leche League : des femmes pour l'allaitement maternel (1956-2004)
}

\section{Gilza SANDRE-PEREIRA}

\section{(2) OpenEdition}

1 Journals

\section{Édition électronique}

URL : https://journals.openedition.org/clio/1462

DOI : $10.4000 /$ clio. 1462

ISSN : 1777-5299

Éditeur

Belin

\section{Édition imprimée}

Date de publication : 1 avril 2005

Pagination : 174-187

ISBN : 2-85816-781-8

ISSN : $1252-7017$

Référence électronique

Gilza SANDRE-PEREIRA, « La Leche League: des femmes pour l'allaitement maternel (1956-2004) », Clio. Histoire, femmes et sociétés [En ligne], 21 | 2005, mis en ligne le 01 juin 2007, consulté le 23 avril 2022. URL : http://journals.openedition.org/clio/1462 ; DOI : https://doi.org/10.4000/clio.1462

Ce document a été généré automatiquement le 23 avril 2022.

Tous droits réservés 


\title{
La Leche League : des femmes pour l'allaitement maternel (1956-2004)
}

\author{
Gilza SANDRE-PEREIRA
}

1 L'allaitement maternel semble retenir de plus en plus l'attention des femmes et les associations qui s'efforcent d'encourager les mères à allaiter sont nombreuses et actives. La CoFAM - Coordination française pour l'Allaitement Maternel ${ }^{1}$ - répertorie actuellement plus de vingt associations qui se déploient en plus de 180 groupes. On peut citer entre autres Solidarilait, Action pour l'Allaitement, Galactée, Info Allaitement, Écoute Lait. La Leche League est cependant la plus connue et la plus active sur le territoire, avec ses 140 groupes en métropole auxquels s'ajoutent ceux de Guadeloupe, Martinique et île de la Réunion. L'analyse suivante s'appuie sur des entretiens avec une représentante de la Leche League France et avec des femmes qui participent aux réunions, ainsi que sur la consultation de quelques publications de l'association'2.

Un réseau international

2 Née aux États-Unis en 1956, fondée par sept mères de famille de la banlieue de Chicago, la Leche League (LLL) est l'association de mères la plus connue et la plus répandue dans le monde. Son objectif est d'informer et de soutenir les femmes qui désirent allaiter leurs bébés à travers une aide personnelle « de mère à mère ». Cette méthode est liée à l'idée que seules les mères ayant fait elles-mêmes l'expérience de nourrir au sein sont capables d'aider une femme à réussir son allaitement et son maternage. Les groupes s'organisent localement grâce à des mères bénévoles - les animatrices - qui ont eu une histoire réussie d'allaitement au sein et partagent la «philosophie » maternaliste de la League $^{3}$.

3 Selon l'historienne américaine Lynn Y. Weiner qui s'est entretenue avec quelques-unes de ses fondatrices, la naissance de l'association et son idéologie s'inscrivent dans le cadre des débats sur le genre ayant eu lieu dans l'après-guerre aux États-Unis. Les fondatrices de la League sont ainsi décrites comme « catholiques romaines, blanches, de classe moyenne, qui se sont connues à travers l'activisme dans le Christian Family Mouvement, et à travers un intérêt commun pour l'accouchement naturel et 
l'allaitement maternel $»^{4}$. Le lien avec le Christian Family Mouvement (CFM), association œcuménique, apparaît clairement dans la méthode proposée pour l'organisation des groupes. La forme des réunions reprend celle utilisée originellement par le CFM : des petits groupes de discussion sur la religion, fonctionnant en tant que «cellule d'action » où des égaux se soutiennent mutuellement. Les animatrices de la League, qui doivent être au courant des derniers développements de la recherche scientifique sur l'allaitement maternel, reçoivent une formation initiale et continuent de se perfectionner par le biais de congrès et de documentation spécialisée. Aux États-Unis, la League est réputée être la meilleure source d'information spécialisée sur le maternage, dépassant l'United States Children's Bureau ${ }^{5}$.

4 Alors que le projet initial était celui d'un groupe local, la croissance de la League aux États-Unis est rapide sur l'ensemble du territoire : 43 groupes de mères en 1961, 1.260 en 1971, environ 3.000 en 1976 et quelques 17.000 animatrices en 1981, tandis que le taux d'allaitement maternel passe d'environ $20 \%$ à $60 \%$ du milieu des années 1950 au milieu des années 1980. La League devient internationale en 1964, avec la création de six groupes dans des pays autres que les États-Unis. Cette "exportation» est due en particulier au déplacement de familles américaines à l'étranger. Le caractère international de cette association se confirme en 1981, quand l'association se met à travailler en collaboration avec l'UNICEF pour la promotion de l'allaitement maternel dans plusieurs pays, notamment du Tiers Monde. La Leche League International a aujourd'hui un statut de conseiller dans le Bureau des ONG auprès des Nations Unies. En 1991, lorsque l'OMS et l'UNICEF ont conçu le projet de l'Hôpital Ami du Bébé (The Baby-Friendly Hospital Initiative), la League était présente, ayant participé avec d'autres organisations à la formation de la WABA (World Alliance for Breastfeeding Action, Alliance Mondiale pour l'Action en Allaitement), un réseau international pour protéger, soutenir et promouvoir l'allaitement maternel. Selon le rapport 2002-03, la League existe aujourd'hui dans 64 pays, son action de mère à mère s'étendant à travers un réseau de près de 41.000 animatrices accréditées. Le succès et la croissance de la League dans le monde sont vraiment étonnants, d'autant que l'association, son idéologie et ses méthodes n'ont pas beaucoup changé durant toutes ces années.

Une philosophie et une méthode : le «naturel » de la maternité

5 Le discours de la League privilégie une approche naturaliste de la maternité. Cependant, il ne s'agit pas - comme cela a été le cas dans le discours des XVIII ${ }^{\mathrm{e}}$ et XIX ${ }^{\mathrm{e}}$ siècles - de penser le rôle maternel dans une approche moraliste où la maternité serait un symbole de la famille associé au modèle chrétien de la Vierge et de l'enfant Jésus. Lynn Y. Weiner montre que, pour les fondatrices de la League, mère et enfant sont pensés comme "symboles de la nature et de la simplicité ", en contrepoint d'une époque centrée sur le scientifique et l'industriel. En effet, la " philosophie » de l'association est fondée sur cette affirmation: «Le maternage par l'allaitement est la façon la plus naturelle et efficace de comprendre et satisfaire les besoins du bébé ». Ce discours redonne à la femme une autonomie face à ce qui est pensé comme l'intrusion dans la famille des experts scientifiques et du pouvoir médical; remettant ainsi en question le "maternage scientifique ", il a été entendu de façon positive par beaucoup de femmes américaines ${ }^{6}$. Les mouvements sociaux des années 1960 et 1970 donnent une amplitude accrue à ce discours. Comme l'ont montré d'autres enquêtes, les mères participantes de la League attribuent aux comportements individualistes et consuméristes modernes le 
fait que les mères, globalement, « refusent leur instinct de maternage - un instinct qui est particulièrement fort quand les mères allaitent $»^{7}$.

D'une certaine façon, c'est cette idée du caractère «naturel» du maternage qui donne sens à la méthode d'action fondée sur cette idée simple : rien ne remplace l'expérience d'une mère. L'objectif affiché des réunions est donc de contribuer à réveiller chez les femmes un instinct maternel endormi par l'usage abusif de la technologie ${ }^{8}$. Ainsi, une animatrice de la League, en dépit de ses connaissances scientifiques sur l'allaitement, ne se présente pas en tant que spécialiste, mais en tant que mère. Et c'est en tant que telle qu'elle cherche à démontrer le rôle d'expert que la nature a donné à la mère en ce qui concerne son enfant. Dans le numéro 19 d'Allaiter Aujourd'hui, une publication de LLL France, le message est explicite : « Observez votre enfant, mettez-vous sur sa longueur d'onde, suivez votre cœur, c'est vous qui savez ce qui est bon pour lui ${ }^{9}$ ». C'est cette conception d'un vécu de la maternité plus proche du naturel qui attire certaines femmes à la League. Pour ces femmes, l'allaitement au sein fait effectivement partie d'une recherche du «naturel», qui s'exprime aussi dans d'autres choix de leur vie comme l'accouchement à la maison, l'utilisation de couches en tissu pour leurs bébés, la consommation de produits biologiques ou faits-maison plutôt que des produits industriels, etc.

La Leche League en France : une secte?

7 La Leche League est arrivée en France en 1975 par le biais d'une animatrice américaine venue vivre à Paris. Au début, les réunions se déroulaient en anglais et étaient destinées plutôt aux femmes américaines ${ }^{10}$. Trois ou quatre groupes se sont constitués, de manière informelle, au cours des années 1970, avec des animatrices américaines expatriées ; mais la naissance officielle de la Leche League France date de 1979, lorsque l'association a été déclarée sous la loi de 1901. Sa croissance est plus modeste qu'aux États-Unis : 25 animatrices en 1989, 200 en 1999. Selon les données de 2004, LLL France a aujourd'hui plus de 238 animatrices accréditées et 133 stagiaires. L'association est présente dans toutes les régions françaises à travers 140 antennes locales ${ }^{11}$.

Des Françaises interviewées dans le cadre de notre étude racontent qu'elles ont connu l'association après un échec lors d'un premier allaitement. C'est le cas de Michèle, 24 ans, deux enfants, mariée, infirmière, qui a sevré son enfant car le pédiatre disait qu'elle n'avait plus de lait : "Alors j'étais très déçue... Bon... J'ai fait comme ça. Tout le monde m'a dit : Ah ! Tu n'as plus de lait, c'est normal. Et voilà ! Alors j'ai tout arrêté ». Lors d'une nouvelle grossesse, elle a trouvé le téléphone de la Leche League dans un magazine pour femmes enceintes et a appelé ; soutenue par d'autres mères du groupe, son expérience d'allaitement a été tout à fait différente: «Je suis contente d'avoir rencontré cette association ».

Il y a également des femmes qui cherchent un soutien dès le premier enfant. C'est l'expérience racontée par Raphaëlle, 31 ans, ex-commerciale dans la mode (elle a arrêté de travailler après la naissance de sa fille). Une amie lui avait parlé des réunions de la League et Raphaëlle a tenu à s'y rendre pour « voir » d'autres femmes qui allaitent :

Je me suis rendue compte, quand j'étais enceinte, que... quand je disais aux gens 'moi, je vais allaiter', j'avais toutes sortes de remarques : 'si tu y arrives...', 'si tu a du lait', 'si ton lait est bon'... enfin, tous ces trucs. Et puis, en fait, c'est là que je me suis rendue compte que dans notre société on n'est plus habitué à allaiter, quoi !

Raphaëlle tenait à allaiter parce qu'elle a toujours considéré l'allaitement comme une pratique naturelle et familière : sa mère l'a allaitée, dans sa famille les femmes le font, 
et elle trouve belle cette image de l'allaitement. Ce témoignage pose donc la question des modèles sociaux d'alimentation du nourrisson. On observe pourtant en France que le modèle d'allaitement au sein est plutôt faible : quelques-unes de mes informatrices m'ont dit n'avoir jamais vu une femme allaiter devant elles. Une association dont la raison d'être est l'allaitement au sein peut donc sembler plutôt étrange aux yeux des Français. Cette "étrangeté » permet peut-être de comprendre que la Leche League ait parfois une image négative, voire soit associée à une secte.

11 Claude Didierjean-Jouveau, ancienne présidente de LLL France, actuelle Rédactrice en chef de la Revue Allaiter Aujourd'hui et responsable des Relations Publiques de l'association, raconte à ce propos l'expérience d'une sage-femme qui a suivi des formations LLL pour professionnels de santé et qui est devenue ensuite Consultante en Lactation $^{12}$. Invitée à faire une présentation sur l'allaitement maternel pour un groupe de pédiatres, cette sage-femme avait tenu un discours très proche de celui de la League, discours bien accepté par les médecins présents. Mais dès qu'elle mentionna le nom de Leche League, il y eut « une levée de boucliers".

Pour Claude Didierjean-Jouveau, il est difficile de préciser les raisons de ce rejet, mais elle avance quelques explications possibles. Au début des années 1980, quand il n'y avait que cinq ou six animatrices, elles présentaient un profil « un peu en marge de la société ", étant pour la plupart macrobiotiques, ayant accouché à la maison, ne vaccinant pas, faisant l'école à domicile... Des choix qui pouvaient être, éventuellement, "perçus comme des trucs de secte». Aujourd'hui, le profil des animatrices LLL en France est très divers et inclut des femmes exerçant une activité professionnelle. Ensuite, le discours tenu par la League, lors de ses débuts en France, ne pouvait avoir d'écho dans le milieu médical, au moment où l'accouchement devenait de plus en plus médicalisé. Selon Yvonne Knibiehler en effet, 98,5 \% des femmes accouchaient en 1974 en milieu hospitalier, dans une politique de sécurisation des naissances qui eut des effets sur les pratiques concernant l'alimentation du nourrisson ${ }^{13}$. Aujourd'hui, le discours des médecins a évolué et s'est beaucoup rapproché de celui de la League, au sujet, par exemple, de l'allaitement à la demande, de la tétée précoce et de la non utilisation de biberons de complément. Enfin, les réticences françaises peuvent se comprendre à travers l'histoire de cette femme accusée par son mari d'être enrôlée dans une secte, alors qu'elle souhaitait poursuivre l'allaitement au sein de son enfant de cinq mois. Cette femme n'agissait pas différemment de ce que les experts scientifiques - ANAES $^{14}$ incluse - recommandent en matière d'allaitement maternel mais, souligne Mme Didierjean-Jouveau, le décalage entre informations anciennes et connaissances nouvelles par le biais de la Leche League pouvait donner l'impression "qu'elle avait subi un lavage de cerveau ".

13 Ces explications sont plausibles mais on peut faire quelques autres hypothèses. D'une part, la faible information des Français concernant l'allaitement maternel. Le manque de formation des professionnels de santé sur l'allaitement au sein, les routines dans les maternités, la non-information des mères, les discours et les actes contradictoires ont été mis en évidence lors de la Quatrième Rencontre de Pédiatrie Pratique qui a eu lieu à Paris en janvier $2000^{15}$. Ajoutons à cela l'ambiguïté propre aux publicités des industriels $\mathrm{du}$ lait pour nourrisson et celle des magazines pour femmes enceintes. De plus, les politiques gouvernementales françaises concernant l'allaitement maternel sont encore balbutiantes. Même si l'allaitement est cité comme le troisième objectif spécifique du Programme National Nutrition Santé (PNNS), proposé en 2001 pour une durée de cinq 
années, la France reste un des rares pays européens à ne pas avoir de Comité National pour l'Allaitement maternel.

D'autre part, doit être envisagée la question du travail féminin, qui alimente un débat à l'intérieur de la League elle-même. Il n'existe pas de données officielles, mais Mme Didierjean-Jouveau est d'accord pour dire que la plupart des animatrices ne travaillent pas en dehors du foyer, au moins provisoirement. Or, le non-engagement des femmes dans la vie professionnelle peut être considéré «hors-norme » dans un modèle social où le travail des femmes est socialement valorisé, en particulier en France dont le taux compte parmi les plus hauts de l'Europe (INSEE, 2004). Pour suivre les recommandations de la League, mère et enfant doivent rester ensemble, ce qui éloigne les femmes des activités hors foyer. Selon L. Y. Weiner, c'est là le paradoxe, et même le piège de l'idéologie maternaliste de l'organisation: «un chemin par lequel simultanément on promeut l'autonomie de la femme et restreint les rôles féminins ». Cependant, il faut considérer que cette question du travail féminin a connu une évolution non négligeable dans le discours de la Leche League depuis cinquante ans.

La question du travail féminin

Au-delà d'une grande permanence dans le discours idéologique, des inflexions peuvent être notées. L'insertion des femmes sur le marché du travail, par exemple, ne faisait pas partie des discussions ou des publications de l'association jusqu'aux années 1970. Dans l'édition de 1981 du Manuel de la League - The Womanly Art of Breastfeeding (L'Art de l'Allaitement Maternel) -, cette question est traitée d'une manière assez négative : la seule justification pour qu'une mère travaille hors du foyer est l'impératif économique (" if at all possible, don't ${ }^{16}$ ) et les animatrices sont instruites à décourager les mères de reprendre leur travail. Dans l'édition de 1987 en revanche, un chapitre traite des mères qui travaillent et la League s'est mise à distribuer un Guide pour aider les femmes à maintenir l'allaitement après la reprise d'activité. Au début des années 1990 cependant, la position de la League reste encore ambiguë. Selon une animatrice américaine, le discours tendant à la conciliation maternage / travail est «l'encouragement sans applaudissement $»^{17}$. Il faut néanmoins considérer que les résultats sur ce point précis peuvent varier beaucoup d'un pays à l'autre.

16 La question du travail féminin est, en effet, très importante quand on parle de l'allaitement maternel. Dans notre étude menée au Brésil et en France, elle apparaît à plusieurs reprises, aussi bien dans les entretiens que dans les discussions sur des sites Internet concernant les mères. Pour certaines femmes, concilier travail et allaitement au sein semble impossible; il faut donc choisir. D'autres s'organisent pour concilier les deux et s'en accommodent plus ou moins bien. Dans tous les cas, le choix est souvent difficile. L'idée qu'il faut arrêter d'allaiter pour reprendre une activité professionnelle est fréquemment exprimée; d'autres prennent un congé ou arrêtent de travailler pour continuer d'allaiter. On peut penser qu'il est plus facile pour une femme de choisir de rester chez elle quand elle n'a pas une véritable carrière professionnelle et que son travail ne représente qu'un complément peu important au budget familial mais il est difficile de dire que cela correspond à une réalité sociale globale. Selon plusieurs études, les taux d'allaitement maternel sont plus importants chez les femmes diplômées de l'enseignement supérieur que chez les femmes ouvrières ou employées ${ }^{18}$. Il est possible que parmi les femmes qui choisissent un congé parental, certaines le fassent en dépit des pertes que ce choix peut induire sur le plan professionnel. C'est le 
cas de Raphaëlle contrainte de beaucoup voyager pour son travail et dont le choix est mal compris par l'entourage :

Là on était d'accord, avec mon ami, là dessus, que... on faisait un enfant ce n'était pas pour que quelqu'un d'autre l'élève, qu'on la fasse courir à la crèche le matin, que... qu'elle fasse ses premiers pas avec quelqu'un d'autre... On a vraiment envie de s'en occuper. Et donc moi j'ai choisi de ne pas travailler. (...) Ne pas travailler, en France c'est dévalorisant. (...) Et maintenant que je leur dis que je reste à la maison pour m'occuper du bébé... ils ne comprennent pas ! Comme si j'avais mieux à faire à l'extérieur, alors que non, je n'ai pas mieux à faire. Ceux qui prêtent attention à la réussite sociale, ils ne comprennent pas. Pour eux, devenir femme au foyer, c'est le pire des trucs!

17 On peut dire ainsi qu'il existe aujourd'hui une pression sociale pour que les femmes reprennent une activité salariée, ce qui n'était pas le cas quelques décennies auparavant. Choisir autrement, c'est nager à contre courant ; un courant d'autant plus fort qu'il est en même temps extérieur et intérieur à l'individu. En effet, pour faire ce choix, la femme doit parfois reconsidérer des valeurs qu'elle tenait pour intangibles, non sans doutes et hésitations :

Là ça va parce que, bon, moi j'ai un petit peu d'économie et puis je touche encore le chômage. C'est vrai que j'ai du mal à l'imaginer si je gagne plus rien de tout... Je sais pas. C'est bête, hein !, mais... ça me gênerait que ce soit... que ce soit mon ami qui travaille et qui ramène... Enfin... que ce soit lui qui doive assumer tout, ça me gênerait.

Concilier allaitement maternel et vie professionnelle devient ainsi et de plus en plus une vraie question pour beaucoup de femmes. Le discours actuel de la League ne peut plus l'ignorer. Dans les publications de LLL France on lit, par exemple, sur le Feuillet $n^{\circ}$ 02 intitulé "Allaitement, Travail et Séparation: Tirer son lait», des informations pratiques pour les femmes qui allaitent et qui travaillent. De son côté, LLL International réalise actuellement un projet nommé «Her Story - An International Perspective on Working and Breastfeeding ${ }^{19}$, en collaboration avec d'autres organisations de soutien à l'allaitement maternel. Il s'agit d'élaborer un ouvrage sur la situation, au plan mondial, des femmes qui travaillent et allaitent, pour informer et motiver les mères à continuer d'allaiter après le congé de maternité, et inciter les politiciens et les chefs d'entreprise à leur offrir localement de bonnes conditions. Ce projet de publication est bien le reflet d'une préoccupation nouvelle de l'organisation, qui avance le chiffre de $70 \%$ des femmes travaillant en dehors du foyer dans le monde entier pour l'an 2010. Sur le terrain, dans le suivi des réunions de groupes de LLL France, on observe pourtant que les mères qui travaillent hors foyer ne sont pas encore en majorité. En fait, les inflexions du discours de la League sur le travail féminin reflètent celles des sociétés occidentales industrialisées, avec leurs contradictions et ambiguïtés. Par exemple, dans le Congrès national de LLL France 2001, une session a été consacrée à "Travail et Allaitement » et deux sessions organisées autour de l'ouvrage de Marie-Pascale Delplancq-Nobecourt Oser être mère au foyer.

19 La maternité et, plus spécifiquement, l'allaitement maternel n'ont pas toujours été intégrés aux revendications pour les droits des femmes. La Leche League, cependant, apparaît historiquement dans un cadre social de changement idéologique du rôle féminin, impulsé d'un côté par le féminisme et la participation croissante des femmes au marché de travail et de l'autre côté par les développements scientifiques et technologiques autour de la reproduction, de la naissance et des soins à l'enfant. Dans ce contexte, le discours pragmatique de l'organisation n'est pas opposé à celui des 
féministes. Il anticipe, d'une certaine façon, le mouvement féministe autour de la santé des femmes, qui met en cause la médicalisation de la naissance et des soins des enfants. En ce sens, on pourrait considérer que la League s'aligne plutôt sur l'idée $d^{\prime}$ '«empowerment $»^{20}$. Ainsi, selon ce point de vue, en choisissant d'allaiter son bébé au sein, la femme confirme son autonomie et conteste à la fois la domination masculine, si présente dans le modèle médical, et les intérêts économiques qui promeuvent l'alimentation par biberon de lait en poudre. En revanche, la permanence d'un discours centré sur le « naturel» de la maternité peut se révéler contradictoire face aux efforts des dernières décennies pour casser la subordination des femmes. C'est seulement quand on considère que la maternité est une partie intégrante et indissociable de l'identité féminine pour de nombreuses femmes, et qu'aujourd'hui en France beaucoup d'entre elles échouent dans leur allaitement par manque de soutien, que l'on peut appréhender le succès d'une telle association dont le rôle est aujourd'hui reconnu par le milieu médical ${ }^{21}$.

En dépit de ses ambiguïtés, l'évolution du discours de la Leache League va aujourd'hui dans le sens d'une réconciliation entre maternité et libération des femmes. Elle essaye d'accompagner ainsi les changements en cours dans les sociétés occidentales, tout en gardant sa spécificité, celle du soutien aux femmes qui choisissent d'allaiter.

\section{BIBLIOGRAPHIE}

AGENCE NATIONALE D'ACCRÉDITATION ET D'ÉVALUATION EN SANTÉ, 2002, Allaitement Maternel - Mise en œuvre et poursuite dans les 6 premiers mois de vie de l'enfant - Recommandations, ANAES, Service recommandations et références professionnelles, mai 2002.

BLUM Linda M. and VANDEWATER Elizabeth A., 1993, “'Mother to Mother' : a maternalist organization in late capitalist America », Social Problems, Vol. 40, n³ 3, august, p. 285-300. DANA Nancy and PRICE Anne, 1987, The Working Woman's Guide to Breastfeeding, New York, Meadowbrook. (Distribué par La Leche League)

DIDIERJEAN-JOUVEAU Claude, 1994, «Confiance en soi, comment elle se perd, comment elle se gagne? », Allaiter Aujourd'hui, n 19, p. 7-8, LLL France.

GOJARD Séverine, 1998, Nourrir son enfant : une question d'éducation - Normes savantes, usages populaires et expérience familiale, Thèse de Doctorat de Sociologie, Paris, École des Hautes Études en Sciences Sociales.

INSEE, 2004, Femmes et hommes : regards sur la parité, Paris, INSEE.

KNIBIEHLER Yvonne, 1997, La révolution maternelle depuis 1945, Paris, Perrin.

LA LECHE LEAGUE INTERNATIONAL, page web, <www.lalecheleague.org>.

LA LECHE LEAGUE INTERnAtionaL, 1981, The Womanly Art of Breastfeeding, Franklin Park, Illinois, LLLI.

LA LECHE LEAGUE INTERNATIONAL, 1987, The Womanly Art of Breastfeeding, Franklin Park, Illinois, LLLI. 
LA LECHE LEAGUE FRANCE, 2001, « Allaitement, Travail et Séparation : Tirer son lait », Feuillet n02, octobre, Paris, LLL France.

LAVIOLLE Gisèle, 1998, Une semaine ordinaire dans la vie de LLL France : association d'information et de soutien pour l'allaitement maternel, Mémoire D.U. Santé publique et communautaire, Faculté de Médecine de Nancy (22p.).

LORAS-DUCLAUX Irène, 2000, La promotion de l'allaitement maternel : des déclarations d'intention à la bonne pratique, Conférence présentée à la Quatrième Rencontre de Pédiatrie Pratique, 21 janv., Paris, Palais des Congrès.

WEINER Lynn Y., 1994, «Reconstructing Motherhood: The Leche League in Postwar America », The Journal of American History, Vol. 80, n 4, March, p. 1357-1381.

\section{NOTES}

1. La Coordination française pour l'Allaitement Maternel (CoFAM) est née en 1999 autour de la Semaine Mondiale de l'Allaitement Maternel ; elle est devenue, en mai 2000, une association dite « loi $1901 »$. Cette association rassemble d'autres groupes ou associations et des professionnels de santé intéressés par la promotion de l'allaitement maternel.

2. Les données ici présentées font partie d'une étude comparative sur l'allaitement maternel au Brésil et en France, menée dans le cadre du doctorat en Anthropologie Sociale au Centre d'Anthropologie de Toulouse - École des Hautes Études en Sciences Sociales / Université de Toulouse II, sous la direction d'Agnès Fine.

3. Selon les informations données par Mme Claude Didierjean-Jouveau, responsable des Relations Publiques de LLL France, pour devenir une animatrice, la femme doit, de préférence, appartenir à un groupe de La Leche League et son expérience d'allaitement doit être telle que le préconise la League : « le bébé a été nourri avec le lait de sa mère jusqu'à ce qu'il manifeste son besoin pour d'autres aliments (ce qui se manifeste en général dans le milieu de sa première année pour un bébé né à terme et en bonne santé). Si le bébé est sevré, il a été allaité environ un an et le sevrage s'est déroulé en respectant les besoins du bébé. » (www.lalecheleague.org). Des femmes qui n'ont aucun groupe proche mais qui ont cette expérience d'allaitement au sein peuvent aussi suivre la formation pour être animatrices LLL.

4. Weiner $1994: 1360$.

5. Semblable au Comité national de l'enfance en France.

6. Weiner $1994: 1358$.

7. Blum et Vanderwater, $1993: 295$.

8. Cette question est l'objet de plusieurs travaux dans le domaine de l'endocrinologie. À ce propos, LLLFrance fait référence surtout aux études du gynécologue français, établi en Angleterre, le Dr. Michel Odent.

9. Didierjean-Jouveau $1994: 8$.

10. D'ailleurs, selon Mme Didierjean-Juveau, responsable des Relations Publiques de LLL France, il existe toujours à Paris des réunions pour des femmes anglophones.

11. www.lllfrance.org. La Leche League n'a pas une présence aussi marquée au Brésil qu'en France. On retrouve cette association dans la seule ville de Maceió, au Nord-Est du pays. En revanche, il existe d'autres groupes locaux de soutien à l'allaitement 
maternel, la plupart organisés par des professionnels de la santé. Il existe aussi quelques groupes semblables à LLL, fondés sur le soutien de mère à mère.

12. Un Consultant en Lactation est celui qui a obtenu le diplôme américain "Certified Lactation Consultants ", un diplôme non universitaire conféré par le bureau associatif IBLCE - International Board of Lactation Consultant Examiners (Bureau International d'Examinateurs des Consultants en Lactation) moyennant la réussite à l'examen proposé annuellement par ce Bureau dans plusieurs pays. L'Association Française des Consultants en Lactation est l'organisme responsable pour l'examen de l'IBLCE en France et le pays comptait 25 consultants en lactation diplômés, selon les données de 2003.

13. Knibiehler 1997.

14. ANAES - Agence Nationale d'Accréditation et d'Évaluation en Santé.

15. Loras-Duclaux 2000.

16. "si c'est possible, ne le faites pas ", LLLI $1981: 271$.

17. Blum et Vanderwater $1993: 289$. Ce paragraphe s'appuie aussi sur Dana et Price 1987.

18. Gojard 1998 ; ANAES, 2002.

19. Détails sur le site Internet de LLL International : <http://www.lalecheleague. org/ donor/story.html>.

20. Le terme "empowerment » peut être défini comme " appropriation ou ré-

appropriation du pouvoir ", et se traduit, sur le plan individuel, par des notions comme confiance en soi, initiative et contrôle, mais aussi compétence personnelle.

L'» empowerment » suppose l'acquisition de nouvelles compétences et renvoie donc aux capacités intellectuelles (savoir et savoir-faire) et à la possibilité de faire des choix libres et éclairés.

21. Comme le montre l'enquête de Gisèle Laviolle, près de la moitié des femmes qui font appel à LLL France ont appris son existence par des professionnels de santé (Laviolle 2002). De son côté, l'ANAES recommande le type d'intervention de mère à mère (ANAES, 2002).

\section{RÉSUMÉS}

La Leche League est une association dont le but est d'aider les femmes à réussir l'allaitement au sein de leurs enfants. Cet article analyse les permanences et les changements de son discours depuis 1956. Il souligne les manières opposées dont elle est perçue et le rôle qu'elle peut jouer aujourd'hui dans les débats sur la maternité en France.

This article deals with La Leche League association, whose goal was to help women in breastfeeding. More specifically, it focuses on continuities and change in the League's discourse over its nearly fifty years of existence. It underlines the opposing ways this association was perceived and the role that it can play today in the debates on motherhood in France. 
INDEX

Mots-clés : allaitement maternel, La Leche League, maternage, travail féminin

\section{AUTEUR}

\section{GILZA SANDRE-PEREIRA}

Gilza SANDRE-PEREIRA est enseignante à l'Institut de Nutrition Josué de Castro (Université Fédérale de Rio de Janeiro, Brésil) et doctorante en Anthropologie Sociale à Toulouse (dir. Agnès Fine). Ses travaux portent notamment sur l'allaitement maternel. Elle a publié en français : « Les politiques pour l'allaitement maternel au Brésil » (Les Dossiers de l'Obstétrique, 2003);

«L'allaitement maternel et l'identité féminine. Le Brésil et la France » (Maternité et parentalité, dir. Y. Knibiehler et G. Neyrand, ENSP, 2004). 